\title{
Nano-catalysts with magnetic core: sustainable options for greener synthesis
}

\author{
Rajender S Varma
}

\begin{abstract}
Author's perspective on nano-catalysts with magnetic core is summarized with recent work from his laboratory. Magnetically recyclable nano-catalysts and their use in benign media is an ideal blend for the development of sustainable methodologies in organic synthesis. Water or polyethylene glycol (PEG) provides good medium to perform such chemical reactions with magnetic nano-catalysts, as this combination adds exceptional value to the overall sustainable process development. In this mini-review, the uses of magnetically recyclable nano-catalysts for a variety of organic reactions are described in conjunction with activation via microwave irradiation.
\end{abstract}

Keywords: Greener synthesis, Nano-catalysis, Magnetic nano-catalysts, Microwave irradiation, Aqueous medium

Chemical developments in the new millennium are now routinely utilizing the concept of "green chemistry" to meet the challenges of protecting the environment and human health while maintaining commercial viability. Green Chemistry is defined as "the utilization of a set of principles that eliminates or reduces the use or generation of hazardous substances in the design, manufacture, and application of chemical products" and emphasizes hazard reduction as the performance criteria while designing new chemical processes. Nanotechnology processes in the recent years have enabled paradigm changing developments in environmental science, medicine and importantly, catalysis. One of the key areas for achieving sustainability is to explore the generation of efficient catalytic processes, via nano-catalysis [1] as a viable option; catalysis has been an integral part of what is now defined more clearly under nanotechnology domain [2].

\section{Nanoparticles-Greener synthesis}

Nanoparticles are the miniscule building blocks for an array of new commercial products and consumer materials in the emerging field of nanotechnology; they are discovered and introduced in the market place at a very fast pace. Nanoparticles can be defined as particulate matter with at least one dimension that is less than $100 \mathrm{~nm}$. The

Correspondence: Varma.Rajender@epa.gov

Sustainable Technology Division, National Risk Management Research

Laboratory, U.S. Environmental Protection Agency, 26 West Martin Luther

King Drive, MS 443, Cincinnati, Ohio 45268, USA commercial interest in nanotechnology has significantly increased with more than US $\$ 9$ billion in investment from public and private sources [3]. Nanoparticles have an exceptionally large surface area to volume ratio, an important trait that is responsible for their widespread advantageous use in catalysis. The nanoscale size and shape imparts unique properties to catalysts because of the structural and electronic changes which differentiates them from the bulk materials. The fine tuning of nanocatalysts, in terms of composition (bimetallic, core-shell type or use of supports), shape and size has accomplished greater selectivity.

In the catalysis arena, the production of engineered nanomaterials is a major breakthrough in material science. A sustained effort has been made to develop eco-friendly strategies to generate these nanomaterials via pathways that use benign reagents rather than the hazardous substances conventionally used. The sustainable strategy for the preparation of nanoparticles has been exemplified by the use of vitamins $B_{1}, B_{2}, C$, and tea and wine polyphenols [4], which function both as reducing and capping agents [5]. This obviates the need to use toxic reducing agents, such as borohydrides or hydrazines. These extremely simple and aqueous green synthetic methods generate bulk quantities of nano-catalysts without the need for large amounts of insoluble templates [6] and have found numerous applications in catalysis $[1,5,7]$.
() Chemistry Central

๑) 2014 Varma; licensee Chemistry Central Ltd. This is an Open Access article distributed under the terms of the Creative Commons Attribution License (http://creativecommons.org/licenses/by/4.0), which permits unrestricted use, distribution, and reproduction in any medium, provided the original work is properly credited. The Creative Commons Public Domain Dedication waiver (http://creativecommons.org/publicdomain/zero/1.0/) applies to the data made available in this article, unless otherwise stated. 


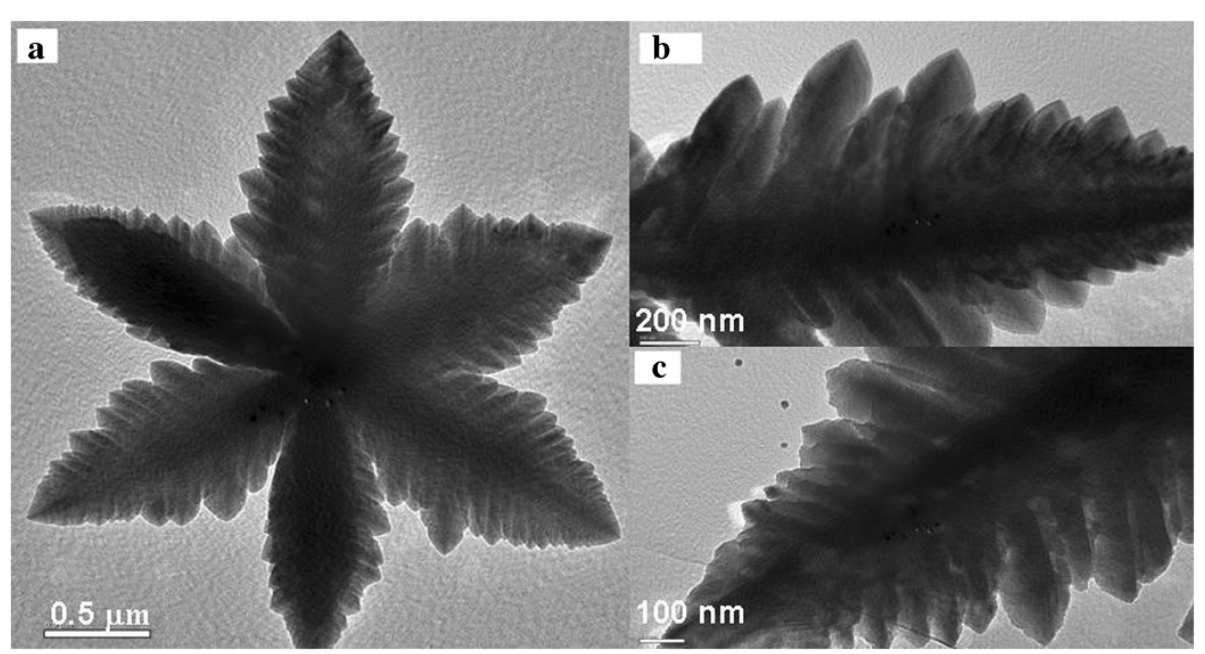

Figure $1 \mathrm{MW}$ Synthesis of dendritic nano-ferrites (micro-pine morphology) from inexpensive starting materials in water without using any reducing or capping reagent agent; panels $\mathbf{b}$ and $\mathbf{c}$ show the well-defined and highly ordered branches. (Reproduced by permission from Royal Society of Chemistry, reference 7).

\section{Alternate energy input- Use of microwave irradiation}

The desired approach to the preparation of uniformly small-sized nano-catalysts may include alternative activation methodology, such as microwave (MW)-, and ultrasonic irradiation and mechanochemical mixing
[8]. The rapid and in-core MW heating has proven a useful method for the synthesis of these metallic nanostructures in solutions $[9,10]$. The valuable application of this method has been established for the preparation of silver $(\mathrm{Ag})$, gold $(\mathrm{Au})$, platinum $(\mathrm{Pt})$, and gold-palladium (Au-Pd) nanostructures [5,11]. MW heating conditions

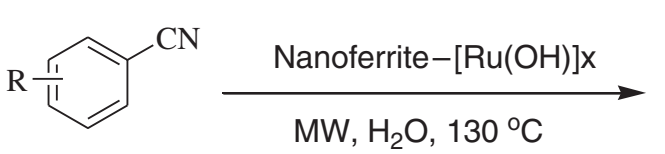

$\mathrm{R}=\mathrm{H}, \mathrm{NO}_{2}, \mathrm{NMe}_{2}, \mathrm{OMe}, \mathrm{Cl}$, aliphatic nitriles etc.<smiles>[R][X]c1ccc(C(N)=O)cc1</smiles>

$(61-88 \%)$

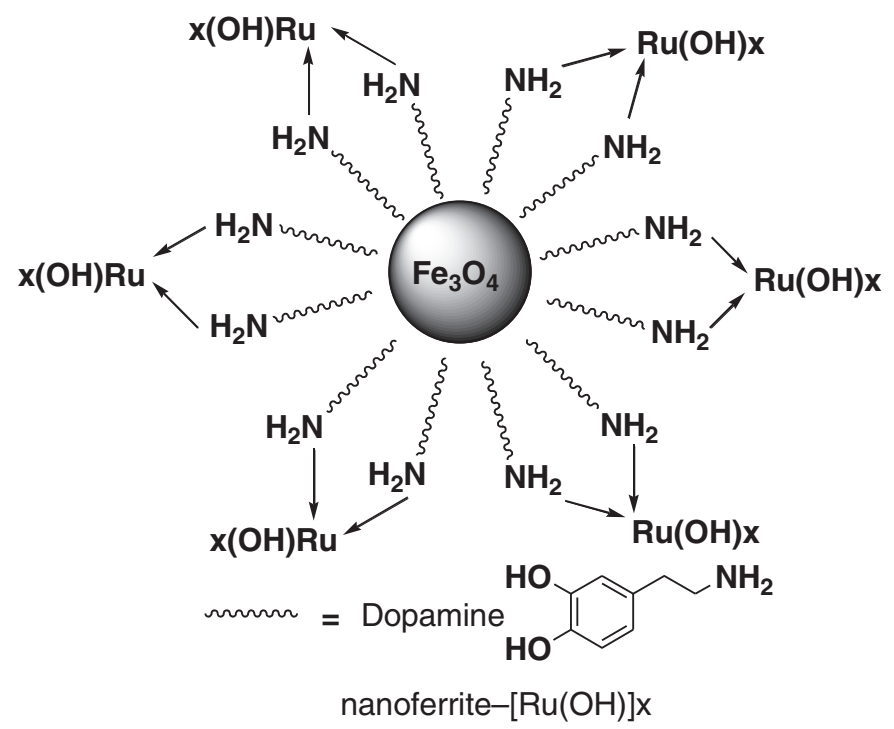

Scheme 1 Nanoferrite-[Ru(OH)]x catalyzed aqueous hydration of nitriles (reproduced by permission from Royal Society of Chemistry, reference 7 ). 


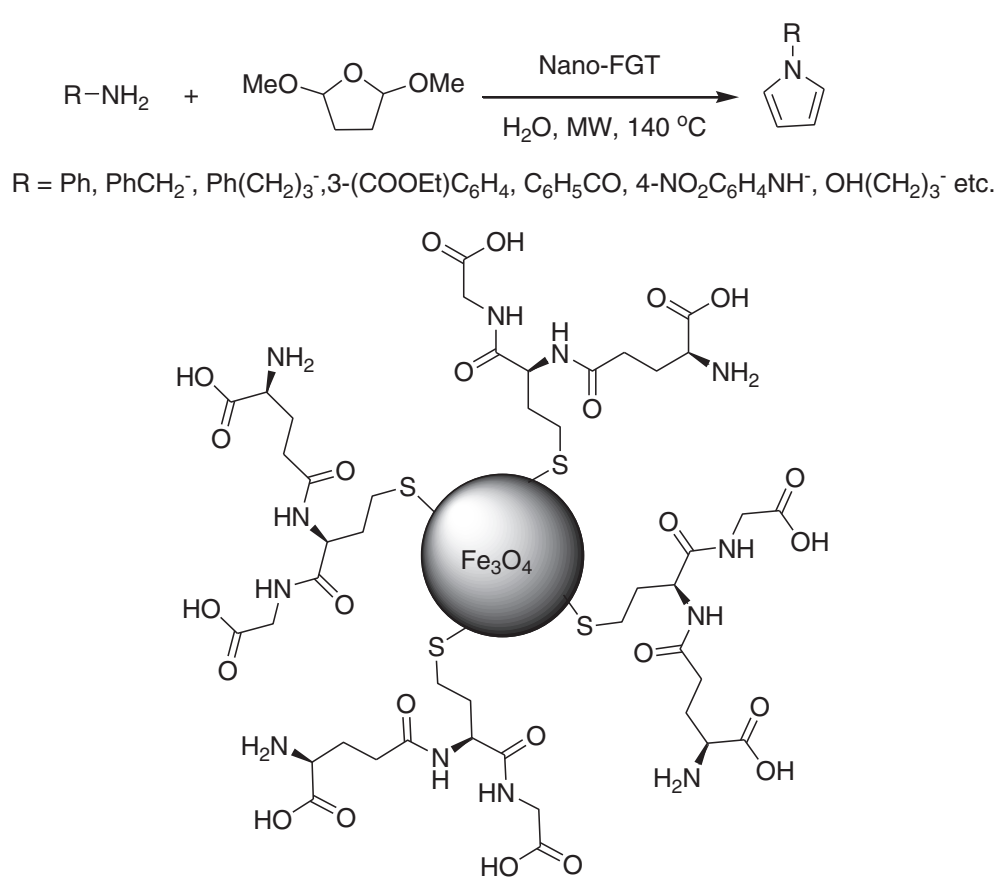

Scheme 2 Nano-FGT-catalyzed Paal-Knorr reactions (reproduced by permission from Royal Society of Chemistry, reference 7).

not only allow the rapid preparation of spherical nanoparticles, but also enables the formation of single crystalline polygonal plates, sheets, rods, wires, tubes, and dendrites as well [12]. Nanostructures of uniformly smaller sizes and with narrower size distributions and a higher degree of crystallization have been obtained using MW heating than those prepared via the conventional oil-bath heating; MW approach allows the greener synthesis of nanomaterials with several desirable features, such as shorter reaction times, better product yields and reduced energy consumption (Figure 1) [7].

Importantly, the strategy encompasses "benign by design" principles and focus on the utilization of renewable resources, if possible $[4,5]$.

\section{Nanoparticles with magnetic core}

To protect the environment and to avoid undue manipulation efforts to purify the product and separation of the catalysts, there is a pressing need to develop methodologies that can facilitate recycle and reuse of these materials at low concentrations and in complex matrices. In this context, iron-based magnetic nanoparticles (MNPs) have been developed that address these needs [13]. These MNPs can be further divided based on the nature of the magnetic core, which can be made up of either reduced species or oxides such as iron oxide NPs $\left(\mathrm{Fe}_{2} \mathrm{O}_{3}\right.$ and $\left.\mathrm{Fe}_{3} \mathrm{O}_{4}\right)$ that has found application in oxidative and coupling reactions $[14,15]$. Such unmodified bare iron oxides comprising $\mathrm{Fe}_{3} \mathrm{O}_{4}$ and $\mathrm{Fe}_{2} \mathrm{O}_{3}$ are found to be active catalysts for the coupling of aldehyde, alkyne, and amine ( $\mathrm{A}^{3}$ coupling) to afford an easy route to propargylamines [16].

The use of MNPs as catalysts in chemical synthesis has been extensively studied in recent years as the recovery of expensive catalysts after their use are some of the salient features in the sustainable process development [17-21]. MNPs coated with benign ligands such as dopamine or glutathione have also been developed and used as heterogeneous catalysts for numerous organic transformations and syntheses [17-21]. The functionalization of the surfaces of nano-sized magnetic materials provides a

$$
\begin{aligned}
& \mathrm{R}_{1} \widehat{\mathrm{Br}}_{+\mathrm{NaN}}+\mathrm{R}_{2}=\frac{\mathrm{MW} 120^{\circ} \mathrm{C}}{\mathrm{H}_{2} \mathrm{O} \text {, Nano-FGT-Cu}} \sim \mathrm{R}_{1} \\
& \mathrm{R}_{1}=\text { Aryl, alkyl, heterocyles } \\
& \mathrm{R}_{2}=\text { Aryl, alkyl, heterocyles }
\end{aligned}
$$

Scheme 3 Nano-FGT-Cu catalyzed 1,3-dipolar cycloadditions reaction (reproduced by permission from Royal Society of Chemistry, reference 7 ). 


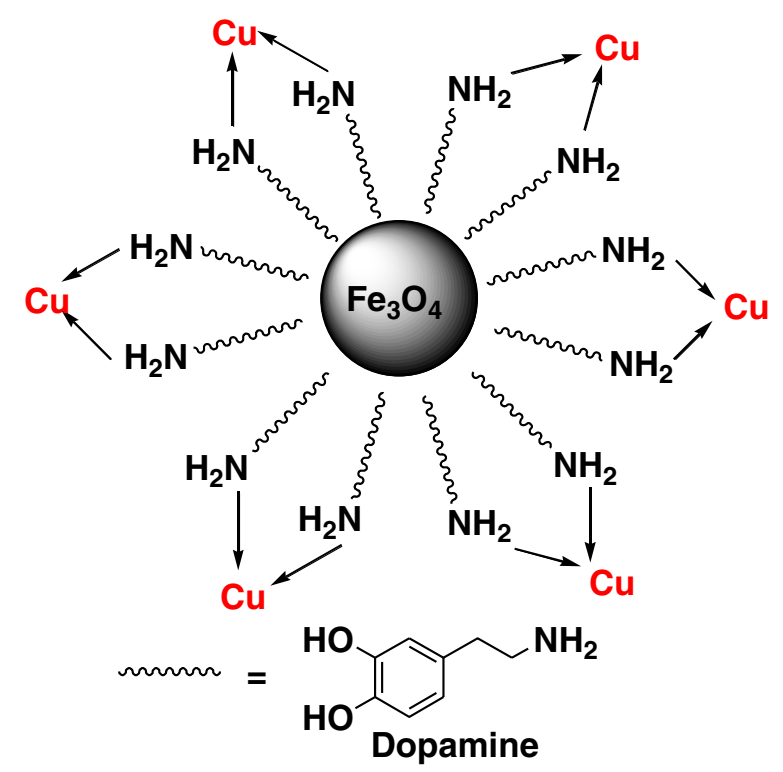

Figure 2 Nano- $\mathrm{Fe}_{3} \mathrm{O}_{4}$-DOPA-Cu catalyst (nano-FeDOPACu) (reproduced by permission from Royal Society of Chemistry, reference 7).

quasi-homogeneous phase in reaction media and acts as a bridge between heterogeneous and homogeneous catalysis thus retaining the relative virtues of both of the systems.

The greener generation of nanoparticles and their ecofriendly applications in catalysis via magnetically recoverable and recyclable nano-catalysts for a variety of oxidation, reduction, and condensation reactions [20-25], has made a tremendous impact on the development of sustainable pathways. This heterogenization of the catalyst in the form of MNPs allows them to be recovered using an external magnet and facilitate their subsequent reuse more efficiently. In view of the reduced size of MNPs in nm range, most of the catalysts surface is accessible for reaction as it provides quasi homogeneous media for the catalysts.

\section{Synthetic modification of magnetic nanoparticles}

The synthetic modification of nanoferrites with dopamine and subsequent anchoring of the metal particles (Scheme 1) on its surface provides numerous opportunities to deploy these nano-catalysts effectively. The hydration of benzonitrile with ruthenium hydroxide on magnetic nano-ferrites transforms it to benzamide in water is an example [22]. The catalyst can be easily separated using an external magnet and after its separation, the clear reaction mixture could be cooled to generate the crystals of benzamides. The complete procedure could be conducted exclusively in aqueous medium that is devoid of organic solvents [22]; the MW-assisted reaction proceeds with high turnover numbers and is attributed to the use of a nanocatalyst.

In an analogous manner, ubiquitous glutathione (GT) molecules have been immobilized on magnetic nanoferrites via their thiol groups [20] and the resulting catalyst (nano-FGT) has been used for the efficient synthesis of a wide variety of aryl, alkyl, and heterocyclic amines (Scheme 2). Such organocatalytic approach enables facile conversion of functionalized amines selectively into the corresponding pyrroles without affecting several sensitive functional groups [25]. This salient feature of immobilization of organocatalysts on MNPs enables their separation magnetically, after completion of the reaction, thus avoiding the cumbersome traditional separation by chromatographic means and solvent usage.

Such nanoparticle-supported and magnetically recoverable organocatalysts help catalyze the synthesis of heterocyclic entities (Paal-Knorr and other reactions) in pure aqueous medium thus precluding the use of toxic organic solvents, even in the work-up stages $[1,7,25]$.

Magnetically separable nano-FGT-Cu catalyst could be used for azide alkyne cycloaddition (AAC) reaction and this general reaction is performed in one-pot via in situ azide generation followed by cycloaddition in aqueous media (Scheme 3) [26].

A similar nano-FeDOPACu bimetallic catalyst (Figure 2) has been deployed in the C-S coupling of aryl halides with thiophenols under MW irradiation conditions [27]; several iodides and bromides (with the exception of aryl chlorides) undergo reaction with thiophenols, affording the corresponding diaryl sulfides (Scheme 4).

The activity of a Fe-Cu bimetallic catalyst can, however, be altered by changing the anchoring ligand for the immobilization of copper nanoparticles on magnetic nano ferrite $\left(\mathrm{Fe}_{3} \mathrm{O}_{4}\right)$ surface. When glutathione is used, the catalyst shows activity for Huisgen cycloaddition [26].

$$
\begin{aligned}
& \mathrm{R}_{1}=\mathrm{H}, \mathrm{Me}, \mathrm{OMe}, \mathrm{Ac}, \mathrm{NO}_{2} \\
& \mathrm{R}_{2}=\mathrm{H}, \mathrm{Cl}, \mathrm{NO}_{2} \\
& \mathrm{X}=\mathrm{I}, \mathrm{Br}
\end{aligned}
$$

Scheme 4 Nano-FeDOPACu catalyzed cross coupling of aryl halides with thiophenols. 


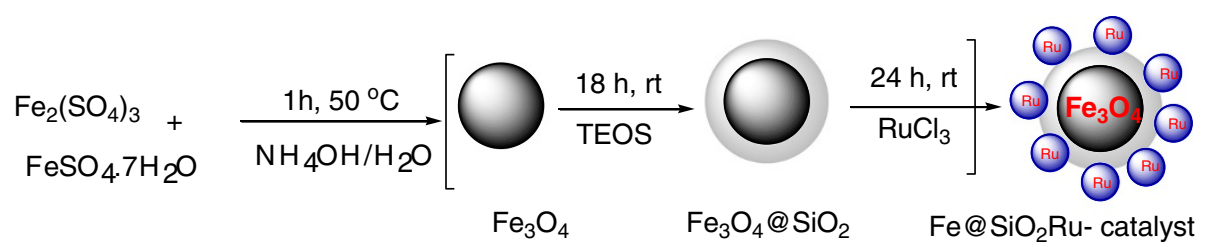

One pot synthesis of nano-Fe@ $\mathrm{SiO}_{2} \mathrm{Ru}$ catalyst

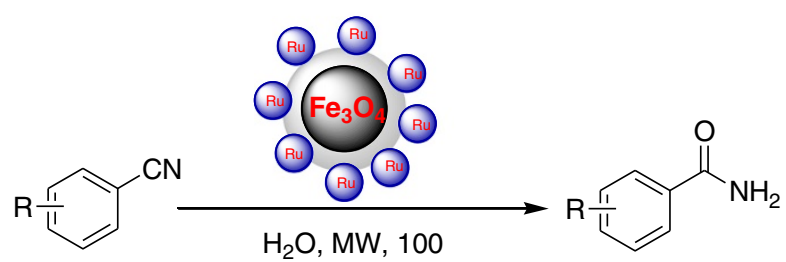

Scheme 5 Hydration of nitriles by nano-Fe@SiO ${ }_{2} \mathrm{Ru}$ catalyst (reproduced by permission from Royal Society of Chemistry, reference 7).

In contrast, when dopamine is used as the anchoring ligand for $\mathrm{Cu}$ nanoparticles, the ensuing catalyst is active for C-S coupling and completely inactive for the Huisgen cycloaddition reactions [28].

A conceptually simple strategy has been advanced for synthetic chemists wherein synthesis of $\mathrm{Fe}_{3} \mathrm{O}_{4} @ \mathrm{SiO}_{2} \mathrm{Ru}$ for the hydration of nitriles in aqueous media occurs
(Scheme 5) via sequential addition of reagents in one-pot to generate and to coat the magnetic particles [29].

Simple mesoporous silica supported iron oxide nanoparticles have been used for the oxidation of alkenes using hydrogen peroxide in water [30]. Styrenes and their substituted derivatives are converted to respective aldehydes in excellent yield (Scheme 6). It is important<smiles>C=Cc1ccccc1</smiles><smiles>O=Cc1ccccc1</smiles>

(95\%)<smiles>O=Cc1ccc(Cl)cc1</smiles>

(90\%)<smiles>O=Cc1ccc(F)cc1</smiles>

(92\%)<smiles>O=Cc1ccc(CCl)cc1</smiles>

(92\%)<smiles>O=Cc1ccc(Br)cc1</smiles>

(90\%)<smiles>O=Cc1ccccn1</smiles>

(95\%)<smiles>[GeH3]</smiles><smiles>COc1ccccc1C=O</smiles><smiles>O=Cc1cccc(Cl)c1</smiles>

$(90 \%)$

(93\%)

Scheme 6 Mesoporous silica supported iron oxide nanoparticles for the oxidation of alkenes using hydrogen peroxide in aqueous medium. 


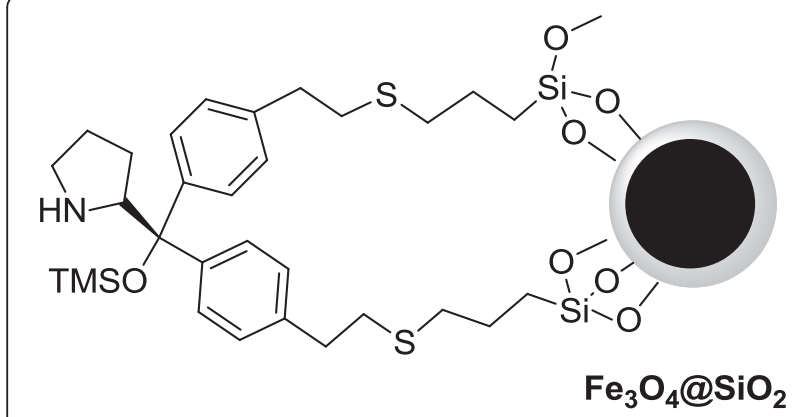

Figure 3 MNP-supported Jørgensen-Hayashi Catalyst.

to note that no other side products or oxidation of aldehydes are observed during the reaction. The optimized protocol is suitable to a range of electron-withdrawing and electron-donating substituents localized in all positions of the aromatic ring.

Supported iron oxide nanocatalysts have been the focus in various important catalytic applications because of their low cost and toxicity, ready availability, and environmentally benign nature $[7,31]$.

\section{Asymmetric synthesis using magnetic nanoparticles}

The utility of MNP supported catalysts in asymmetric synthesis has been illustrated in an example where superparamagnetic nanoparticle-supported $(S)$-diphenylprolinol trimethylsilyl ether has been employed for the asymmetric Michael addition in water [32]; Jørgensen-Hayashi catalyst, ((S)-diphenylprolinol trimethylsilyl ether) on silica superparamagnetic nanoparticle support, (Figure 3), was prepared by a multistep synthetic procedure.

As-synthesized MNP-supported Jørgensen-Hayashi catalyst accomplished the asymmetric Michael addition of enolisable aldehydes to nitroalkenes in water; the corresponding products were obtained in moderate to good yields (up to 96\%), and with good enantioselectivity (up to $90 \%$ ee) (Scheme 7).

Significantly, the reaction was conducted between trans$\beta$-nitrostyrene and propanal in water to deliver high yield of the product (85\%). A variety of nitroalkanes and aldehydes performed well under these optimized reactions conditions and the corresponding products were obtained in good to excellent yields in benign aqueous medium.

Synthetic processes using alternative energy input in combination with nano-catalysts thus shorten the reaction time and that eliminate or minimize side product formation [8]. This concept when used in the syntheses of pharmaceuticals, fine chemicals, and polymers may pave the way towards the greener and more sustainable approach to chemical syntheses. Newer developments on these themes, especially involving benign reaction media such as water and polyethylene glycol (PEG), in conjunction with photo activation [33], MW and ultrasonic irradiation, and/or ball-milling under solvent-free conditions, may help realize sustainable pathways for chemical synthesis and transformations, including the generation of novel nano-catalysts $[7,8]$.

Nano-catalysts, especially those that can be recycled magnetically, are of special value in synthetic domain. The modifications of the surface of MNPs with other nanometals are of interest for catalysis. Such post-synthetic alterations using organic ligands [20], mimicking organocatalysis, allows the adsorption of catalytically active metal nanoparticles, which provide identical or better reactivity than the corresponding homogeneous catalysts $[25,34]$. The enhanced dispersity of the MNPs in common solvents is an added advantage, since it exposes the surface-bound active reaction sites for the reactants in ideal fashion. In foreseeable future, the design of novel magnetically retrievable heterogeneous asymmetric catalysts supported on $\mathrm{Fe}_{3} \mathrm{O}_{4}$ nanoparticle systems will find significant applications in asymmetric hydrogenations [35], asymmetric $\mathrm{C}-\mathrm{C}$ bond formation reactions, and in asymmetric cycloaddition reactions, especially under continuous-flow conditions in microreactors [36].

Bare iron oxide nanoparticles provide ready access to oxidation and oxidative coupling reactions, while the reduced $\mathrm{Fe}(0)$ NPs facilitate dehydrogenation, hydrogenation, couplings and reductive processes. The incorporation of a second metal serves to futher expand the catalytic abilities of Fe and incorporation of oxides on to silica has been utilized for the biodiesel production [37]. Supporting various ligands on the magnetic nanoparticles facilitates synthesis of several chemical entities with sustainable advantages, predominant being the magnetic separation

$$
\begin{aligned}
& \mathrm{R}_{\mathrm{CHO}}^{+} \overbrace{\mathrm{Ar}}^{\mathrm{NO}_{2}} \\
& \text { MNP-JHC (20 mol\%) } \\
& \text { RT, } 72 \text { hrs, } \mathrm{H}_{2} \mathrm{O} \\
& \mathrm{R}_{\mathrm{R}}^{\mathrm{Ar}} \mathrm{NO}_{2} \\
& \text { 53-96 \% }
\end{aligned}
$$

Scheme 7 Aqueous asymmetric Michael addition of aldehydes to nitroalkenes. 
and recyclability aspects [38,39]. Incorporation of copper provides an easy preparation of propargylamines via a multicomponent reaction [40] and asymmetric nanocatalysis is facilitated by support of heterocyclic carbenes as chiral modifiers [41].

Magnetic nanoparticles are garnering special attention in the emerging area of flow chemistry. The dual function of confinement and agitation of the nanoparticle-bound catalyst can be achieved in a reactor by means of a rotating magnetic field, which avoids the potential problems of clogging membranes or filters that are eminent barriers for immobilized catalysts. Reiser et al. have demonstrated this successfully in a close circuit reactor for the asymmetric benzoylation of racemic 1,2-diols using a copper (II)-azabis(oxazoline) catalyst, which had been covalently attached to carbon-coated cobalt nanoparticles [42]; magnetic field induced flow mixing is beginning to make critical impact for handling of slurries and precipitates in modern small footprint flow reactors [43].

\section{Competing interests}

The author declares that he has no competing interest.

\section{Authors' information}

Prof. Rajender S. Varma was born in India (Ph.D., Delhi University 1976). After postdoctoral research at Robert Robinson Laboratories, Liverpool, UK, he was faculty member at Baylor College of Medicine and Sam Houston State University prior to joining Sustainable Technology Division at US Environmental Protection Agency in 1999. He has over 40 years of research experience in management of multi-disciplinary technical programs ranging from natural products chemistry to therapeutics and development of environmentally friendlier alternatives for synthetic methods using microwaves, and ultrasound etc. More recently, he is focused on greener approaches to assembly of nanomaterials and sustainable applications of magnetically retrievable nano-catalysts in benign media. He is member of the editorial advisory board of several international journals and published over 390 scientific papers and has been awarded 12 US Patents.

\section{Acknowledgements}

I wish to thank my collaborators and colleagues, past and present, whose names appear in the reference section for their immense contribution to our efforts in Green and Sustainable Chemistry research program.

Received: 24 February 2014 Accepted: 17 April 2014

Published: 16 May 2014

\section{References}

1. Polshettiwar V, Varma RS: Green chemistry by nano-catalysis. Green Chem 2010, 12:743-754.

2. Astruc D, Lu F, Aranzaes JR: Nanoparticles as recyclable catalysts: The frontier between homogeneous and heterogeneous catalysis. Angew Chem Inter Edn: 2005, 44:7852-7872.

3. Eckelman MJ, Zimmerman JB, Anastas PT: Toward green nano: E-factor analysis of several nanomaterial syntheses. J Ind Ecol 2008, 12:316-328.

4. Baruwati B, Varma RS: High value products from waste: grape pomace extract - a three-in-one package for the synthesis of metal nanoparticles. ChemSusChem 2009, 2:1041-1044

5. Varma RS: Greener approach to nanomaterials and their sustainable applications. Curr Opin Chem Eng 2012, 1:123-128.

6. Nadagouda MN, Speth T, Varma RS: Microwave-assisted green synthesis of silver nanostructures. Acc Chem Res 2011, 44:469-478.

7. Varma RS: Journey on greener pathways: From the use of alternate energy inputs and benign reaction media to sustainable applications of nano-catalysts in synthesis and environmental remediation. Green Chem 2014, 16:2027-2047.
8. Nasir Baig RB, Varma RS: Alternate energy input: mechanochemical, microwave and ultrasound-assisted organic synthesis. Chem Soc Rev 2012, 41:1559-1584.

9. Polshettiwar V, Nadagouda MN, Varma RS: Microwave-assisted chemistry: A rapid and sustainable route to synthesis of organics and nanomaterials. Aust J Chem 2009, 62:16-26.

10. Baghbanzadeh M, Skapin SD, Orel ZC, Kappe CO: Critical assessment of the specific role of microwave irradiation in the synthesis of $\mathrm{ZnO}$ micro- and nano-structure materials. Chem Eur J 2012, 18:5724-5731.

11. Varma RS: Green chemical processes: sustainable nanomaterials from waste agricultural residues and their applications. Speciality Chemicals Magazine 2013, 33:27-28.

12. Polshettiwar V, Baruwati B, Varma RS: Self-assembly of metal oxides into three-dimensional nanostructures: synthesis and application in catalysis. ACS Nano 2009, 3:728-736.

13. Baruwati B, Nadagouda MN, Varma RS: Bulk synthesis of monodisperse ferrite nanoparticles at water-organic interfaces under conventional and microwave hydrothermal treatment and their surface functionalization. J Phys Chem C 2008, 112:18399-18404.

14. Enthaler $S$, Junge $K$, Beller M: Sustainable metal catalysis with iron: from rust to a rising star? Angew Chem Int Edn 2008, 47:3317-3321.

15. Bolm C, Legros J, Le Paih J, Zani L: Iron-catalyzed reactions in organic synthesis. Chem Rev 2004, 104:6217-6254.

16. Zeng TQ, Chen WW, Cirtiu CM, Moores A, Song GH, Li CJ: $\mathrm{Fe}_{3} \mathrm{O}_{4}$ nanoparticles: a robust and magnetically recoverable catalyst for three-component coupling of aldehyde, alkyne and amine. Green Chem 2010, 12:570-573.

17. Baig RBN, Varma RS: Magnetically retrievable catalysts for organic synthesis. Chem Commun 2013, 49:752-770.

18. Gawande MB, Rathi AK, Branco PS, Varma RS: Sustainable utility of magnetically recyclable nano-catalysts in water: applications in organic synthesis. App/ Sci 2013, 3:656-674.

19. Gawande $\mathrm{MB}, \mathrm{Branco} P \mathrm{~S}$, Varma RS: Nano-magnetite $\left(\mathrm{Fe}_{3} \mathrm{O}_{4}\right)$ as a support for recyclable catalysts in the development of sustainable methodologies. Chem Soc Rev 2013, 42:3371-3393.

20. Polshettiwar V, Baruwati B, Varma RS: Magnetic nanoparticle-supported glutathione: A conceptually sustainable organocatalyst. Chem Commun 2009:1837-1839.

21. Baig RBN, Varma RS: Organic synthesis via magnetic attraction: benign and sustainable protocols using magnetic nanoferrites. Green Chem 2013, 15:398-417.

22. Polshettiwar V, Varma RS: Nanoparticle-supported and magnetically recoverable ruthenium hydroxide catalyst: Efficient hydration of nitriles to amides in aqueous medium. Chem Eur J 2009, 15:1582-1586.

23. Polshettiwar $V$, Baruwati B, Varma RS: Nanoparticle-supported and magnetically recoverable nickel catalyst: a robust and economic hydrogenation and transfer hydrogenation protocol. Green Chem 2009, 11:127-131.

24. Luque R, Baruwati B, Varma RS: Magnetically separable nanoferriteanchored glutathione: aqueous homocoupling of arylboronic acids under microwave irradiation. Green Chem 2010, 12:1540-1543.

25. Polshettiwar $V$, Varma RS: Nano-organocatalyst: magnetically retrievable ferrite-anchored glutathione for microwave-assisted Paal-Knorr reaction, aza-Michael addition, and pyrazole synthesis. Tetrahedron 2010, 66:1091-1097.

26. Baig RBN, Varma RS: A highly active magnetically recoverable nano ferrite-glutathione-copper (nano-FGT-Cu) catalyst for Huisgen 1,3-dipolar cycloadditions. Green Chem 2012, 14:625-632.

27. Baig RBN, Varma RS: A highly active and magnetically retrievable nanoferrite-DOPA-copper catalyst for the coupling of thiophenols with aryl halides. Chem Commun 2012, 48:2582-2584.

28. Baig RBN, Varma RS: Copper modified magnetic bimetallic nano-catalysts: ligand regulated catalytic activity. Curr Org Chem 2013, 17:2227-2237.

29. Baig RBN, Varma RS: A facile one-pot synthesis of ruthenium hydroxide nanoparticles on magnetic silica: aqueous hydration of nitriles to amides. Chem Commun 2012, 48:6220-6222.

30. Rajabi F, Karimi N, Saidi MR, Primo A, Varma RS, Luque R: Unprecedented selective oxidation of styrene derivatives using a supported iron oxide nanocatalyst in aqueous medium. Adv Synth Catal 2012, 354:1707-1711.

31. Polshettiwar V, Baruwati B, Varma RS: Nanoparticle-supported and magnetically recoverable nickel catalyst: a robust and economic hydrogenation and transfer hydrogenation protocol. Green Chem 2009, 11:127-131. 
32. Wang BG, Ma BC, Wang Q, Wang W: Superparamagnetic nanoparticle-supported (s)-diphenylprolinol trimethylsilyl ether as a recyclable catalyst for asymmetric Michael addition in water. Adv Synth Catal 2010, 352:2923-2928.

33. Balu AM, Baruwati B, Serrano E, Cot J, Garcia-Martinez J, Varma RS, Luque R: Magnetically separable nanocomposites with photocatalytic activity under visible light for the selective transformation of biomass-derived platform molecules. Green Chem 2011, 13:2750-2758.

34. Varma RS: Greener routes to organics and nanomaterials: sustainable applications of nano-catalysts. Pure \& Applied Chem 2013, 85:1703-1710.

35. Hu A, Yee GT, Lin W: Magnetically recoverable chiral catalysts immobilized on magnetite nanoparticles for asymmetric hydrogenation of aromatic ketones. J Am Chem Soc 2005, 127:12486-12487.

36. Vaddula BR, Varma RS, Gonzalez MA: Supported ruthenium catalysts for sustainable flow chemistry. Curr Org Chem 2013, 17:2268-2278.

37. Suzuta T, Toba M, Abe Y, Yoshimura Y: Iron oxide catalysts supported on porous silica for the production of biodiesel from crude Jatropha oil. J Amer Oil Chem Soc 2012, 89:1981-1989.

38. O' Dalaigh C, Corr SA, Gun'ko Y, Connon SJ: A magnetic-nanoparticle-supported 4-N,N-dialkylaminopyridine catalyst: Excellent reactivity combined with facile catalyst recovery and recyclability. Angew Chem Inter Edn 2007, 46:4329-4332.

39. Li PH, Li BL, An ZM, Mo LP, Cui ZS, Zhang ZH: Magnetic Nanoparticles $\left(\mathrm{CoFe}_{2} \mathrm{O}_{4}\right)$-supported phosphomolybdate as an efficient, green, recyclable catalyst for synthesis of $\beta$-hydroxy hydroperoxides. Adv Synth Catal 2013, 355:2952-2959.

40. Aliaga MJ, Ramon DJ, Yus M: Impregnated copper on magnetite: an efficient and green catalyst for the multicomponent preparation of propargylamines under solvent free conditions. Org \& Biomol Chem 2010, 8:43-46.

41. Ranganath KVS, Kloesges J, Schafer AH, Glorius F: Asymmetric nanocatalysis: $\mathrm{N}$-heterocyclic carbenes as chiral modifiers of $\mathrm{Fe}_{3} \mathrm{O}_{4} / \mathrm{Pd}$ nanoparticles. Angew Chem Inter Edn 2010, 49:7786-7789.

42. Schatz A, Grass RN, Kainz Q, Stark WJ, Reiser O: Cu(II) - azabis(oxazoline) complexes immobilized on magnetic $\mathrm{Co} / \mathrm{C}$ nanoparticles: Kinetic resolution of 1,2-diphenylethane-1,2-diol under batch and continuous-flow conditions. Chem Mater 2010, 22:305-310.

43. Koos P, Browne DL, Ley SV: Continuous stream processing: a prototype magnetic field induced flow mixer. Green Process Synth 2012, 1:11-18.

doi:10.1186/2043-7129-2-11

Cite this article as: Varma: Nano-catalysts with magnetic core: sustainable options for greener synthesis. Sustainable Chemical Processes 2014 2:11.

\section{Publish with ChemistryCentral and every scientist can read your work free of charge \\ "Open access provides opportunities to our colleagues in other parts of the globe, by allowing anyone to view the content free of charge." \\ W. Jeffery Hurst, The Hershey Company. \\ - available free of charge to the entire scientific community \\ - peer reviewed and published immediately upon acceptance \\ - cited in PubMed and archived on PubMed Central \\ - yours - you keep the copyright \\ Submit your manuscript here: \\ http://www.chemistrycentral.com/manuscript/

